\title{
AbaA and WetA govern distinct stages of Aspergillus fumigatus development
}

\begin{abstract}
Correspondence
Jae-Hyuk Yu

jyu1@wisc.edu
\end{abstract}

Received 30 July 2010

Revised 6 October 2010

Accepted 14 October 2010

\section{$\mathrm{Li} \mathrm{Tao}{ }^{1,2}$ and Jae-Hyuk $\mathrm{Yu}^{1}$}

\author{
${ }^{1}$ Departments of Bacteriology and Genetics, University of Wisconsin, Madison, WI 53706, USA \\ ${ }^{2}$ State Key Laboratory of Agrobiotechnology, China Agricultural University, Beijing, PR China
}

The opportunistic human pathogen Aspergillus fumigatus produces a massive number of asexual spores (conidia) as the primary means of dispersal, survival, genome protection and infection of hosts. In this report, we investigate the functions of two developmental regulators, AfuAbaA and AfuWetA, in A. fumigatus. The AfuabaA gene is predicted to encode an ATTS/TEA DNA-binding domain protein and is activated by $A f u B r l A$ during the middle stage of $A$. fumigatus asexual development (conidiation). The deletion of $A f u a b a A$ results in the formation of aberrant conidiophores exhibiting reiterated cylinder-like terminal cells lacking spores. Furthermore, the absence of $A f u a b a A$ causes delayed autolysis and cell death, whereas the overexpression of Afuaba $A$ accelerates these processes, indicating an additional role for AfuAbaA. The AfuwetA gene is sequentially activated by $A f u A b a A$ in the late phase of conidiation. The deletion of Afuwet $A$ causes the formation of defective spore walls and a lack of trehalose biogenesis, leading to a rapid loss of spore viability and reduced tolerance to various stresses. This is the first report to demonstrate that WetA is essential for trehalose biogenesis in conidia. Moreover, the absence of Afuwet $A$ causes delayed germ-tube formation and reduced hyphal branching, suggesting a role of $A f u W e t A$ in the early phase of fungal growth. A genetic model depicting the regulation of conidiation in $A$. fumigatus is proposed.

\section{INTRODUCTION}

Aspergillus fumigatus is a saprophytic soil fungus that causes more infections worldwide than any other mould. Inhalation of asexual spores (conidia) by immunocompromised individuals may lead to germination of spores and growth of the fungal cells in the lung, followed by progression to a serious disease called invasive pulmonary aspergillosis (IPA; reviewed by Cockrill \& Hales, 1999; Dagenais \& Keller, 2009; Rhodes \& Askew, 2010). Recently, despite advances in antifungal therapy, the incidence of IPA has increased significantly and the mortality of patients has risen to approximately $35 \%$ (Ejzykowicz et al., 2009; Gao et al., 2010; Agarwal et al., 2010). As the infectious life cycle of A. fumigatus usually begins with the production of conidia that are small enough to be deposited in the alveoli after being inhaled (Wasylnka \& Moore, 2003), it is important to understand the underlying mechanisms of sporulation in this fungus. This may contribute to the development of new prevention strategies for Aspergillus infection.

Asexual sporulation (conidiation) in A. fumigatus involves the elaboration of a multicellular reproductive structure

Abbreviations: AB, Alamar Blue; ARE, AbaA response element; BRE, BrlA response element; OE, overexpression; PGU, peripheral growth unit.

Supplementary data are available with the online version of this paper. called a conidiophore that requires activity of the key regulator BrlA (Fig. 1a; Mah \& Yu, 2006; reviewed by Yu et al., 2006). The developmental mechanisms in Aspergillus nidulans have been studied intensively and the findings have provided important clues for understanding conidiation in other aspergilli (reviewed by Adams et al., 1998; Ni et al., 2010; see Fig. 1b). In A. nidulans, early conidiophore formation begins with the specification of thick-walled foot cells that branch to form aerial conidiophore stalks (Mims et al., 1988). BrlA, a $\mathrm{C}_{2} \mathrm{H}_{2}$ zinc-finger transcription factor that is expressed during the early phase of conidiation, mediates the switch from polarized stalk elongation to vesicle formation and budding-like cell growth (Adams et al., 1988). Then, a layer of specialized cells (metulae) bud from the vesicle, and two phialides bud from the tip of the metulae and subsequently differentiate into sporeproducing (conidiogenous) cells. Loss-of-function brlA mutants form structures that resemble conidiophore stalks ('bristle' phenotype), except that they grow indeterminately and fail to produce vesicles, metulae, phialides and conidia, indicating that BrlA controls the commencement of conidiophore development (Adams et al., 1988).

The $a b a A$ gene, activated by BrlA during the middle stages of conidiation when metulae and phialides are being actively formed, has been reported to be involved in the differentiation and functionality of phialides. Mutational 
(a)
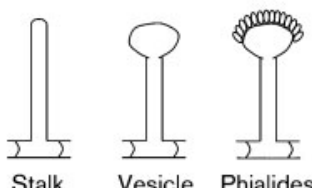

Vesicle Phialides

(c)

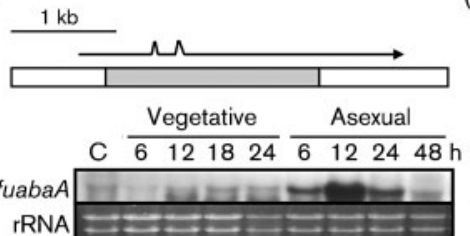

(b)
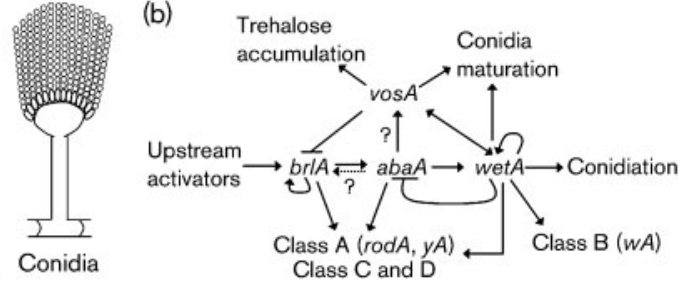

(d)

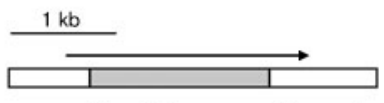

Vegetative Asexual

C $\overline{6121824} \overline{6122448}$ $\mathrm{rRNA}=\mathrm{E}=\mathrm{E}=$

Fig. 1. Background information and expression of AfuabaA and AfuwetA. (a) Formation of the A. fumigatus conidiophore. (b) The current model for downstream regulation of conidiation in A. nidulans (adapted and modified from $\mathrm{Ni} \&$ Yu, 2007). (c) and (d) Northern blot analyses. Levels of AfuabaA (c) and AfuwetA (d) mRNA throughout the life cycle of wild-type $A$. fumigatus (Af293) are presented. Numbers indicate incubation time (h) in liquid MMG (vegetative) and post-asexual developmental (asexual) induction. C represents conidia. Equal loading of total RNA was evaluated by ethidium bromide staining of rRNA. inactivation of $\mathrm{AbaA}$ results in the formation of supernumerary tiers of cells with properties of metulae, not phialides, i.e. the 'abacus' phenotype (Sewall et al., 1990a; Andrianopoulos \& Timberlake, 1994). The wetA gene is activated by AbaA during the late phase of conidiation and plays a role in the synthesis of a crucial conidial wall component, the inner $\mathrm{C} 4$ layer, which makes conidia impermeable and mature (Marshall \& Timberlake, 1991; Sewall et al., 1990b). Together, the $b r l A \rightarrow a b a A \rightarrow$ wetA cascade has been proposed to define a central regulatory pathway that controls temporal and spatial expression of conidiation-specific genes during conidiophore development and spore maturation (Boylan et al., 1987; Mirabito et al., 1989; Ni et al., 2010; see Fig. 1b). Lossof-function mutations in any one of these three genes blocks a specific stage in conidiogenesis, resulting in defective conidiophores that fail to produce mature conidia. Recently, the novel regulator VosA has been identified by a genetic screen. VosA is a multifunctional regulator that acts in concert with the central regulatory genes, couples trehalose biogenesis and conidia maturation, and exerts negative feedback regulation on brlA (Ni \& Yu, 2007; Fig. 1b).

Based on this framework from the study of A. nidulans conidiation, we have been dissecting the mechanisms underlying the regulation of conidiation in A. fumigatus, primarily focusing on characterization of key developmental regulators (reviewed by $\mathrm{Yu}, 2011$ ). Our previous study showed that the role of the core downstream transcription factor BrlA is conserved in both species (Mah \& Yu, 2006; Yu et al., 2006). In the present study, we hypothesize that $A f u A b a A$ and $A f u W e t A$ play distinct roles in A. fumigatus conidiogenesis, and test this by studying sets of AfuabaA and AfuwetA deletion and overexpression $(\mathrm{OE})$ mutants for hyphal growth and conidiogenesis. Our results demonstrate that AfuabaA and AfuwetA direct distinct steps of conidiogenesis, and that the central regulatory pathway is conserved between A. fumigatus and $A$. nidulans. In addition, we further demonstrate that AfuAbaA participates in the regulation of cell death and autolysis, and that $A f u$ WetA is required for normal germtube formation and hyphal branching. A genetic model depicting regulation of conidiogenesis in A. fumigatus is presented.

\section{METHODS}

Strains, media and growth conditions. Escherichia coli $\mathrm{DH} 5 \alpha$ was used for general cloning. Aspergillus strains used in this study are listed in Table 1. The fungal strains were grown on minimal solid or liquid medium $(\mathrm{MM})$ with $0.1 \%$ yeast extract and appropriate supplements (e.g. $1 \mathrm{~g}$ uracil $\mathrm{l}^{-1}+1 \mathrm{~g}$ uridine $\mathrm{l}^{-1}$, MMG; Käfer, 1977) and incubated at $37^{\circ} \mathrm{C}$. For liquid submerged culture, about $1 \times 10^{6}$ conidia $\mathrm{ml}^{-1}$ were inoculated into liquid $\mathrm{MMG}$ and incubated at 250 r.p.m. at $37^{\circ} \mathrm{C}$. To induce AfuabaA overexpression, solid or liquid MM with $100 \mathrm{mM}$ threonine as sole carbon source (MMT) was used. Conidiation induction was performed as described by Seo et al. (2003).

Construction of A. fumigatus strains. The oligonucleotides used in this study are listed in Table $\mathrm{S} 1$ (available with the online version of this paper). Double-joint PCR was used to generate the AfuabaA and AfuwetA deletion constructs (Yu et al., 2004). The deletion constructs containing A. nidulans pyrG (amplified with primer pair OBS8/OBS9) with approximately $1 \mathrm{~kb}$ each of the $5^{\prime}$ and $3^{\prime}$ flanking regions of AfuabaA or AfuwetA were introduced into the recipient strain Af293.1 (AfupyrG1) (Xue et al., 2004). Primer pairs OSG1/OSG2 and OSG7/ OSG8 were used to amplify the $5^{\prime}$-flanking regions of AfuabaA and AfuwetA, respectively. The $3^{\prime}$-flanking regions of the two genes were amplified with primer pairs OSG3/OSG4 (AfuabaA) or OSG9/OSG10 (AfuwetA). Transformation was performed as described by Szewczyk et al. (2006). Solid medium without uracil and uridine was used to select transformants, and deletion candidates were confirmed by PCR followed by enzyme digestion as described previously (Yu et al., 2004). Three AfuabaA and three AfuwetA deletion mutants ( $\triangle A f u a b a A$ and $\triangle A$ fuwet $A$ ) were isolated from 20 and 14 transformants examined, respectively. The alcA(p)::AfuabaA construct was generated by inserting $2518 \mathrm{bp}$ of the AfuabaA ORF (amplified using OLT3 and OLT4) between the KpnI and NotI sites of pNJ25 (Shin et al., 2009b), giving rise to pLT8. Multiple AfuabaA OE strains were generated by transforming Af293.1 with pLT8, where a single copy of alcA(p):: AfuabaA is integrated into the AfupyrG locus. OE candidates were isolated and confirmed by PCR and Northern blot analyses. To generate complemented strains, the AfuabaA(p)::AfuabaA and $A$ fuwetA(p)::AfuwetA constructs amplified with OLT13/OLT14 
Table 1. Aspergillus strains used in this study

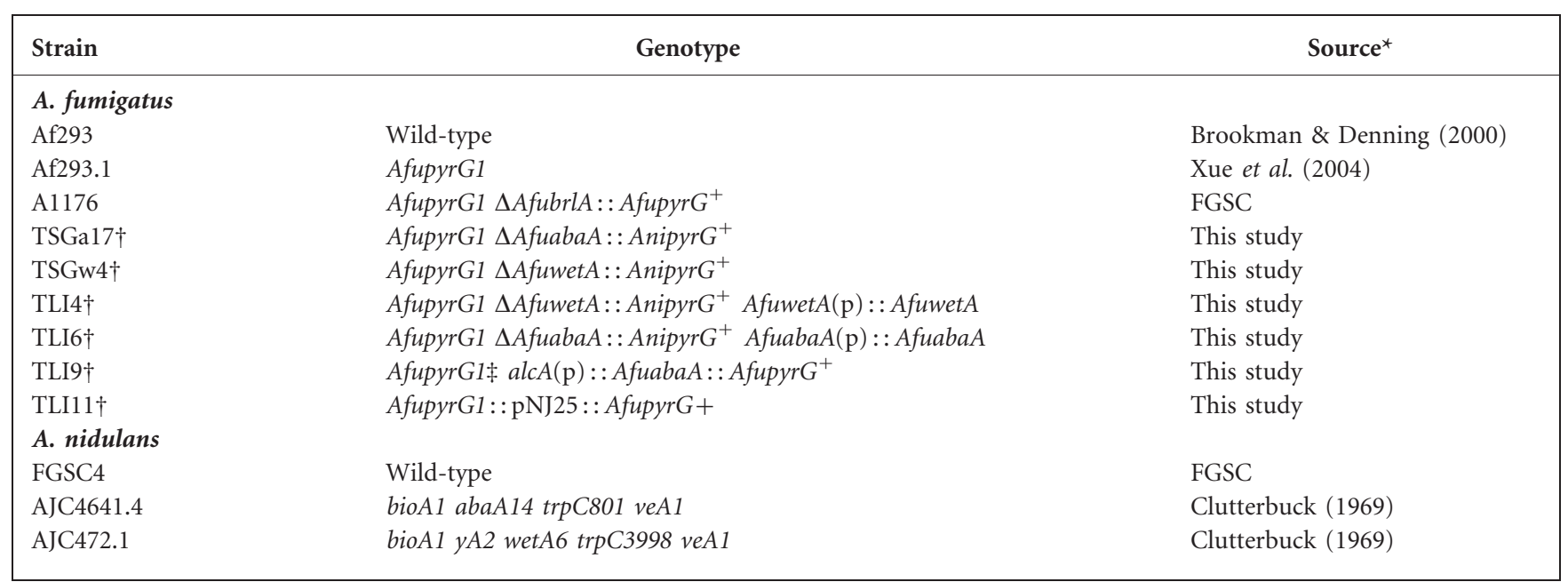

${ }^{\star}$ FGSC, Fungal Genetics Stock Center, School of Biological Sciences, University of Missouri, Kansas City, MO 64110, USA.

$\dagger$ Multiple isogenic strains (all behaved identically).

\$The 3/4 AfupyrG marker in pNJ25 causes targeted integration at the AfupyrG1 locus.

(AfuabaA), and OLT15/OLT16 (AfuwetA), were cloned into pUCH2-8 (HygR), resulting in pLT9 and pLT10, respectively. The AfuabaA(p):: AfuAbaA : :HygR and AfuwetA(p) :: AfuWetA : HygR amplicons were introduced into strains TSGa17 (AfupyrG1 $\triangle$ AfuabaA::Anipyr $G^{+}$) and TSGw4 (AfupyrG1 $\triangle$ AfuwetA::AnipyrG ${ }^{+}$), respectively. Six AfuabaA(p):: AfuAbaA (e.g. TLI6 in Table 1, designated C' AfuabaA in this study) and five $A f u w e t A(p)::$ AfuWetA (e.g. TLI4, designated $C^{\prime}$ AfuwetA) strains exhibiting wild-type (WT) phenotype were isolated and confirmed.

Nucleic acid isolation and manipulation. Genomic DNA isolation, total RNA preparation and Northern blot analyses were carried out as described previously (Yu et al., 2004). Total RNA was isolated from conidia and mycelia collected at various time points. Briefly, $10^{8}$ conidia from relevant strains were inoculated into $100 \mathrm{ml}$ liquid MMG in $250 \mathrm{ml}$ flasks and incubated at $37{ }^{\circ} \mathrm{C}, 250$ r.p.m. For vegetative growth phases, samples were collected at designated time points from liquid submerged cultures. For asexual developmental induction, 18 h-old vegetatively grown mycelia were harvested and transferred to solid MMG (Seo et al., 2006) and samples were collected at the indicated time points. All samples were squeeze-dried, quick-frozen in liquid $\mathrm{N}_{2}$ and stored at $-80{ }^{\circ} \mathrm{C}$ until required for RNA isolation. For total RNA isolation, around $10 \mathrm{mg}$ mycelium was homogenized using a Mini Bead beater in the presence of $1 \mathrm{ml}$ Trizol reagent (Invitrogen) and $0.6 \mathrm{ml}$ silica/zirconium beads (Biospec). RNA extraction from ground hyphae was performed according to the manufacturer's instructions (Invitrogen). A Hybond-N membrane (Amersham) was used for blotting nucleic acids after separation of $10 \mu \mathrm{g}$ total RNA in $1.1 \%$ agarose gel containing $3 \%(\mathrm{v} / \mathrm{v})$ formaldehyde. DNA probes were prepared by amplification of the coding regions of individual genes (AfubrlA, AfuabaA, AfuwetA, AfuvosA and AfurodA) using the appropriate primer pairs with Af293 genomic DNA as template (Table S1).

Confirmation of AfuabaA and AfuwetA ORFs. PCRs were performed to amplify the ORFs of AfuabaA and AfuwetA using the UniZAP A. fumigatus cDNA library as template (Reyes et al., 2006; kindly provided by Dr G. S. May, University of Texas MD Anderson Cancer Center). The sequencing primers OLT59 and OLT60 (forward primers for the AfuabaA ORF), OLT57 and OLT58 (reverse primers for the AfuabaA ORF), OLT63 (forward primer for the AfuwetA ORF), and OLT61 and OLT62 (reverse primers for the AfuwetA ORF) were used. Direct sequencing analyses of the PCR products led us to verify the introns of the AfuabaA and AfuwetA genes by comparing the cDNA sequences with the ORF sequences.

Determination of mycelial mass and cell viability. Dry weight was determined as described previously (Yamazaki et al., 2007; Shin et al., 2009a). Cell viability was determined by the percentage reduction in Alamar Blue $(\mathrm{AB})$. Briefly, the $\mathrm{AB}$ assay reagent (AbD Serotec) was placed into each test well of a 24-well plate (Nunc) containing $1 \mathrm{ml}$ fresh medium and $0.5 \mathrm{ml}$ individual cultures, at a final amount equal to $10 \%$ of the volume in the well. After $6 \mathrm{~h}$ incubation at $37^{\circ} \mathrm{C}$, the absorbance of the contents of each well was measured at 570 and $600 \mathrm{~nm}$. The percentage reduction of $\mathrm{AB}$ was calculated as described previously (Al-Nasiry et al., 2007; Shin et al., 2009a; Tao et al., 2010). The results are representative of triplicate samples of individual cultures.

Spore viability test. To check the viability of spores, fresh conidia from 2-day-old cultured WT (Af293), $\triangle$ AfuwetA and complemented strains on solid MMG were collected and spread onto solid MMG $\left(10^{5}\right.$ spores per plate) and incubated at $37{ }^{\circ} \mathrm{C}$. Then, conidia from 2-, 5-, 10- and 20-day-old cultures were collected, approximately 150 (expected number) conidia were inoculated onto solid MMG and incubated at $37{ }^{\circ} \mathrm{C}$ until colonies appeared. Survival rate (measured in triplicate for each sample) was calculated as the ratio of the number of growing colonies to the expected number of spores inoculated.

Trehalose assay and stress tolerance test. Trehalose was determined as described by d'Enfert \& Fontaine (1997), Fillinger et al. (2001) and $\mathrm{Ni} \& \mathrm{Yu}$ (2007) with a slight modification. Two-dayold spores $\left(2 \times 10^{8}\right)$ were washed with $\mathrm{ddH}_{2} \mathrm{O}$ three times,

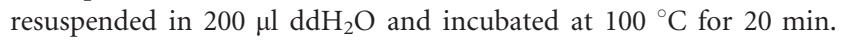
The suspension was then centrifuged for $10 \mathrm{~min}$ at $11000 \mathrm{~g}$, and supernatant containing trehalose was collected. Fifty microlitres of supernatant was mixed with $50 \mu \mathrm{l} 0.2 \mathrm{M}$ sodium citrate ( $\mathrm{pH}$ 5.5) and samples were incubated at $37{ }^{\circ} \mathrm{C}$ for $8 \mathrm{~h}$ with or without $3 \mathrm{mU}$ trehalase (Sigma, T8778). The amount of glucose liberated by the activity of trehalase was assayed using a glucose (GO) assay kit 
(Sigma, GAGO-20) and converted into trehalose per conidium (measured in triplicate). Each sample without trehalase treatment served as a negative control. To examine thermal tolerance, WT, mutant and complemented strain conidia were incubated at $50{ }^{\circ} \mathrm{C}$ for $0,1,2.5$ and $5 \mathrm{~min}$. To examine oxidative tolerance, $\mathrm{WT}$, mutant and complemented strain conidia were treated with varying concentrations $(0,0.025,0.05,0.1$ and $0.2 \mathrm{M})$ of $\mathrm{H}_{2} \mathrm{O}_{2}$ and incubated for $10 \mathrm{~min}$ at room temperature ( $\mathrm{Ni} \& \mathrm{Yu}, 2007)$. Osmotic tolerance of WT, mutant and complemented strain conidia was examined by inoculating conidia on $M M G$ with different concentrations $(0,0.2$, $0.5,1$ and $1.5 \mathrm{M}$ ) of $\mathrm{NaCl}$. For each treatment, the spores were inoculated onto solid MMG and incubated at $37{ }^{\circ} \mathrm{C}$ for 2 days. Colony numbers were counted and calculated as a percentage of the untreated control.

Microscopy. Photomicrographs were taken by using a Zeiss Axioplan 2 microscope with AxioVision digital imaging software (Zeiss). Culture plate and Northern blot photographs were taken using a SONY DSC-F828 digital camera. TEM was performed as described by Ni \& Yu (2007).

\section{RESULTS}

\section{Identification and expression of AfuabaA and AfuwetA}

Our previous study (Yu et al., 2006) identified AfuAbaA (EAL88194, $60 \%$ identity, 74\% similarity with AbaA in A. nidulans) and AfuWetA (EAL89470, 58\% identity, $68 \%$ similarity) through genome searches (Nierman et al., 2005). Here, we further confirmed the ORFs of AfuabaA and AfuwetA by PCR amplification of the coding regions from a cDNA library followed by sequence analyses (see Methods). In summary, the AfuabaA ORF is composed of $2518 \mathrm{bp}$ with three exons and two introns, and is predicted to encode a 797 aa-length protein containing an ATTS/ TEA DNA-binding domain (see Fig. S1a, available with the online version of this paper; Andrianopoulos \& Timberlake, 1991). The AfuwetA ORF comprises 1701 bp with no intron, and is predicted to encode a 566 aa-length protein with a conserved C-terminal domain (see Fig. S1b).

Levels of AfuabaA and AfuwetA transcripts throughout the life cycle were examined by Northern blotting. AfuabaA and AfuwetA genes were highly expressed during conidiation. AfuabaA mRNA started to accumulate at $6 \mathrm{~h}$ postdevelopmental induction, reached the highest level at $12 \mathrm{~h}$ and decreased at $48 \mathrm{~h}$ (Fig. 1c). Accumulation of AfuwetA mRNA was observed at $12 \mathrm{~h}$ post-developmental induction, reached the highest level at $48 \mathrm{~h}$ and was also observed in conidia (Fig. 1d).

\section{AfuAbaA and AfuWetA control distinct stages of conidiogenesis}

To investigate the functions of AfuabaA and AfuwetA, deletion mutants were generated and their phenotypes were examined. Colonies and micrographs of the $\triangle A$ fuabaA and $\triangle A$ fuwetA mutants are shown in Fig. 2(a), and transmission electron micrographs of $a b a A$ and wet $A$ mutant conidiophores in both A. fumigatus and A. nidulans are shown in Fig. 2(b, c). These results indicate that while WT and complemented strains produce a large number of conidia, the $\triangle A f u a b a A$ and $\triangle A f u w e t A$ mutants exhibit aberrant conidiation phenotypes. The $\triangle A$ fuabaA mutant formed defective conidiophores that failed to generate conidia. The $\Delta$ AfuwetA mutant produced colourless conidia and formed droplets of liquid on the surface of the colony. As shown in Fig. 2(b, c), when WT strains developed normal conidia, loss of function of $a b a A$ in both species leads to the formation of aberrant conidiophores exhibiting reiterated cylinder-like terminal cells (elongated phialides) without conidia, indicating that AfuAbaA is essential for differentiation and functionality of A. fumigatus phialides as conidiogenous cells. Furthermore, TEM of the wetA mutants in both A. nidulans and A. fumigatus revealed that wetA conidiophores exhibited the inter-connected conidia phenotype, i.e. incomplete conidial separation and maturation (Fig. 2b, c), implying that $A f u$ WetA plays an essential role in the completion of conidial maturation.

\section{A role of AfuAbaA in cell death and autolysis}

Previous studies reported that $A$. nidulans $a b a A$ also functions in the regulation of autolysis (Emri et al., 2008). To investigate a potential role for $A f u A b a A$ in autolysis and cell death, aberrant $\triangle A f u a b a A$ conidiophores were inoculated into liquid MMG $\left(\sim 10^{4}\right.$ conidiophores $\left.\mathrm{ml}^{-1}\right)$, and the morphology and dry weight of mycelium were examined. Microscopic analyses revealed that the most distal cells of the aberrant $\triangle A f u a b a A$ conidiophores were capable of apical hyphal growth, whereas the fractions of cylinder-like elongated phialides were not able to undergo vegetative growth (Fig. 3a). Determination of dry weight indicated that the absence of AfuabaA caused a delay in the normal reduction of hyphal mass seen in WT (Fig. 3b). Moreover, even after 5 days incubation, when WT and complemented strains exhibited clear hyphal disintegration, the $\triangle A f u a b a A$ mutant still retained its intact and compact hyphal morphology (Fig. 3b). To quantify mycelium fragmentation, the numbers of fragmented hyphae per 100 microscopic fields were counted. The values of WT, $\triangle A f u a b a A$ and complemented strains were $401.43,72.00$ and 363.08 , respectively, demonstrating an approximately fivefold reduction in fragmentation levels in the $\triangle A f u a b a A$ mutant. These results indicate that $A f u A b a A$ is necessary for normal progression of autolysis.

We then examined whether AfuAbaA plays a role in cell death by determining percentage $A B$ reduction, which corresponds to the activity of living cell mitochondria. While both WT and complemented strains displayed dramatically reduced cell viability at day 4 , no reduction in cell viability was observed in the $\triangle A f u a b a A$ mutant (Fig. $3 c$ ). Furthermore, whereas WT and complemented strains displayed a complete loss of cell viability at days 5 and 6 , the $\triangle A f u a b a A$ mutant retained a certain level of viability 
(a)

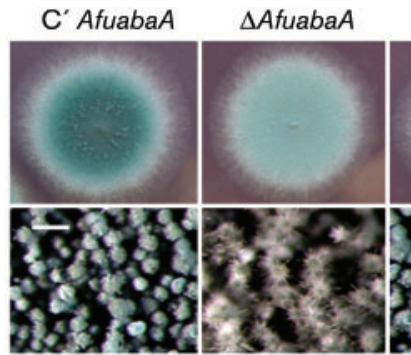

WT

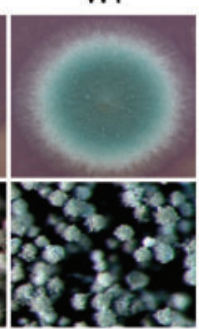

$\triangle$ AfuwetA

C'AfuwetA

(b)

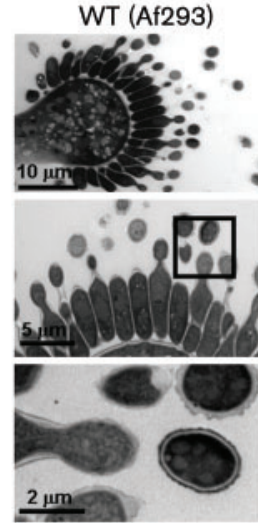

$\triangle$ Afuaba $A$
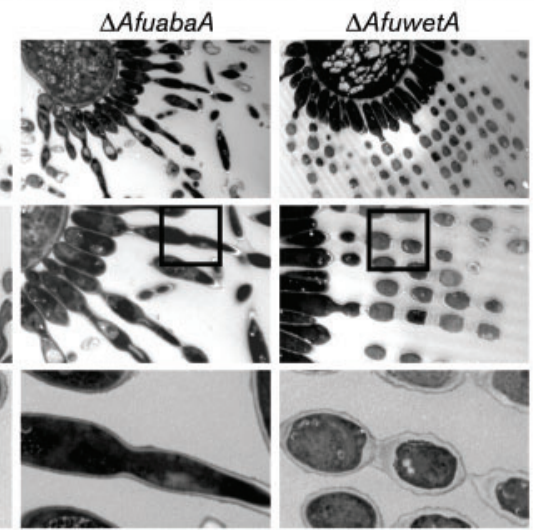

(c)

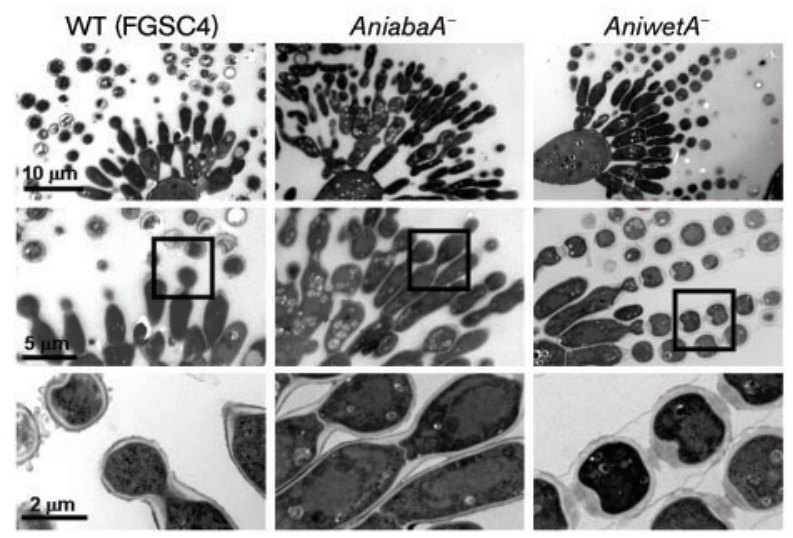

Fig. 2. Requirement for $A f u A b a A$ and $A f u W e t A$ in distinct steps of conidiation. (a) Phenotypes of WT (Af293), $\triangle$ AfuabaA (TSGa17), $\triangle$ AfuwetA (TSGw4) and complemented strains TLI6 (for AfuabaA=C' AfuabaA) and TLI4 (for Afuwet $A=\mathrm{C}^{\prime}$ Afuwet $A$ ) grown on solid MMG at $37{ }^{\circ} \mathrm{C}$ for 3 days. Bar, $100 \mu \mathrm{m}$. (b, c) Effects of $a b a A$ and wet $A$ mutations on conidiogenesis in A. fumigatus (b) and $A$. nidulans (c). TEM images of 2-day-old conidiophores or conidia of the following strains are shown: $A$. fumigatus: WT (Af293), $\triangle$ AfuabaA (TSGa17), $\triangle$ AfuwetA (TSGw4); $A$. nidulans: WT (FGSC4), aba $A^{-}$ (AJC4641.4), wetA- (AJC472.1). Note the cylinder-like terminal cells formed by the $\triangle A f u a b a A$ mutant and the defective conidia produced by the $\triangle$ Afuwet $A$ mutant. even at day 6, indicating that $A f u A b a A$ is required for controlled progression of cell death.

To further confirm the role of $A f u A b a A$ in autolysis and cell death, we tested the effects of overexpression of AfuabaA. As AfuAbaA plays a positive role, upon induction in MMT, OEAfuabaA caused accelerated hyphal disintegration and a dramatic reduction in mycelial mass. As shown in Fig. 4(a, b), while the control strain exhibited normal levels of hyphal extension and conidiation, OEAfuabaA resulted in clear hyphal disintegration and complete lysis at $72 \mathrm{~h}$. Furthermore, on day 2 the OEAfuabaA strain exhibited clear acceleration of cell death compared to the control strain (Fig. 4c), corroborating the idea that AfuAbaA functions positively in autolysis and cell death.

In addition, we found that OEAfuabaA caused adverse effects on hyphal growth and development. First, hyphal tip growth stopped when AfuabaA was overexpressed (Fig. $4 \mathrm{a}, \mathrm{b})$. Second, OEAfuabaA strains exhibited reduced levels of conidiation when grown on solid (Fig. 4d) or in liquid
(Fig. 4a) MMT. To quantify the conidiation level, the number of conidia $\mathrm{mm}^{-2}$ of control and OEAfuabaA strains grown in solid MMG $+0.5 \%$ yeast extract (MMGY) or MMT $+0.5 \%$ yeast extract (MMGT) was counted. When grown in inducing MMTY, the OEAfuabaA strain formed a lower number of conidia at any position of the colony than that produced by the control strain (Fig. 4d), whereas no phenotypic differences were observed when they were grown in MMGY. These results suggest that overexpression of AfuabaA inhibits both hyphal extension and conidiation, and that proper expression of AfuabaA is crucial for normal growth and development in $A$. fumigatus.

\section{Complex roles of AfuWetA}

As AfuWetA plays an essential role in the completion of the conidial wall, we hypothesized that the absence of AfuwetA may affect viability and/or integrity of conidia. To test this, the abilities of 2-, 5-, 10- and 20-day-old WT, $\Delta$ AfuwetA 
(a)
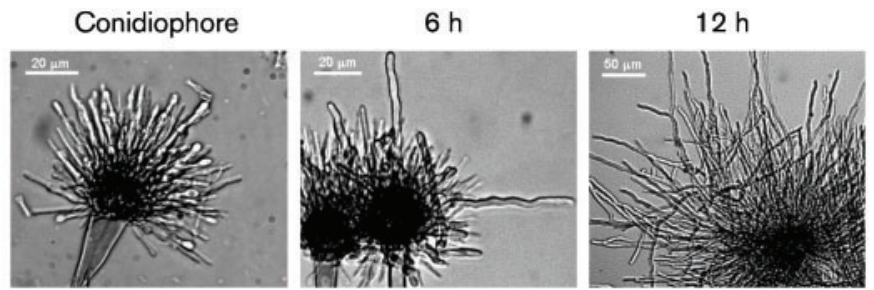

(b)
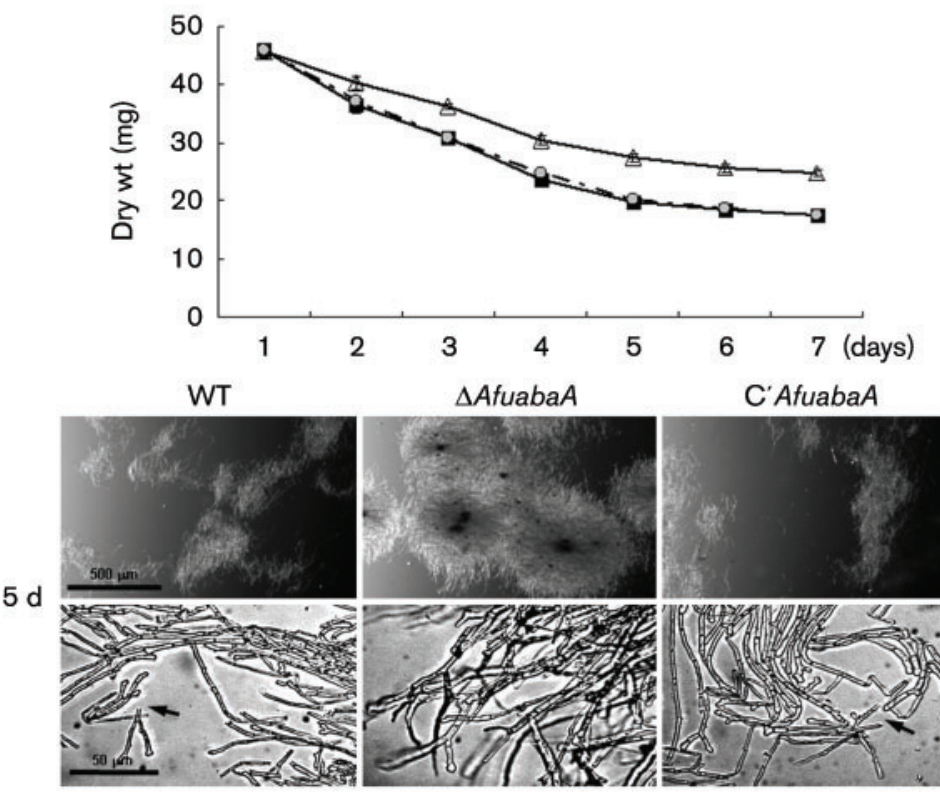

$\triangle$ AfuabaA
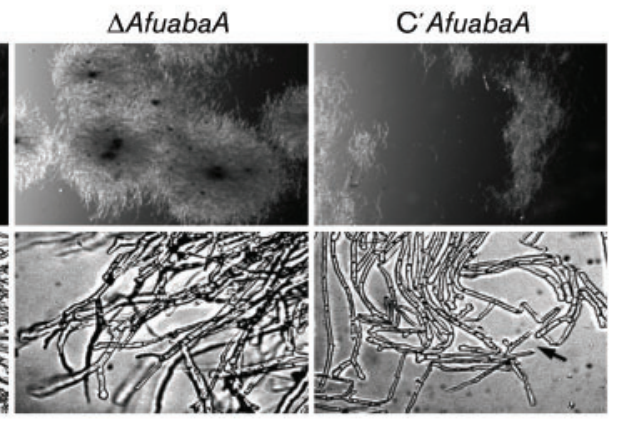

(c)

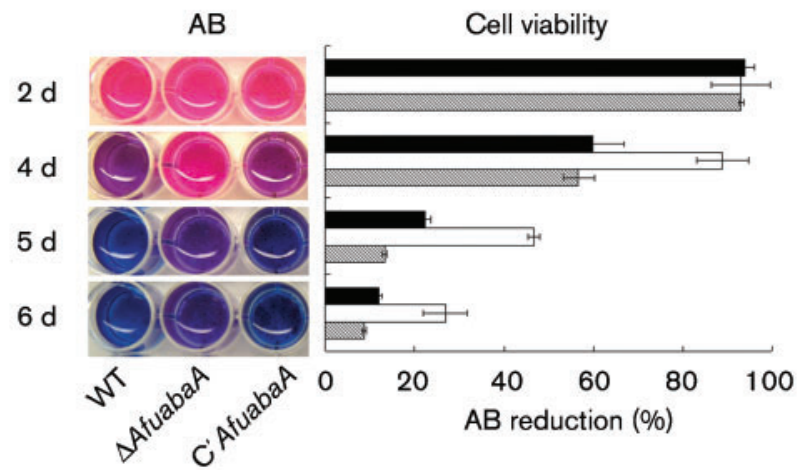

Fig. 3. The role of $A f u A b a A$ in autolysis and cell death. (a) $\triangle$ AfuabaA conidiophores $(0 \mathrm{~h})$ were inoculated into liquid MMG and incubated at $37{ }^{\circ} \mathrm{C}, 250$ r.p.m. Photomicrographs were taken at 6 and $12 \mathrm{~h}$. Note that cylinder cells underwent vegetative growth starting from $6 \mathrm{~h}$ incubation. (b) Mycelial morphology and dry weight of WT (Af293, $\mathbf{\square}), \triangle$ AfuabaA (TSGa17, $\Delta$ ) and complemented (TLI6, O) strains grown in liquid submerged culture. Note that, compared to WT and complemented strains, the $\triangle$ AfuabaA mutant exhibited delayed hyphal disintegration. Arrows indicate the hyphal fragmentation observed in WT and complemented strains. (c) Cell viability of WT (black bars), $\triangle$ AfuabaA (white bars) and complemented strains (hatched bars) determined by $A B$ reduction rates. Data are representative of triplicates of individual cultures. Note that at days 4 and 5 , the $\triangle$ Afuaba $A$ mutant showed high levels of cell viability. and complemented strain conidia to generate colonies were compared. As shown in Fig. 5(a), the $\Delta$ AfuwetA mutant exhibited dramatically reduced viability starting from day 10, while WT and complemented strain conidia maintained high viability until day 20. Moreover, TEM studies revealed that, even on day 2 , about $20 \%$ of the $\Delta$ AfuwetA conidia appeared to lack cytoplasm and exhibited sheared conidial walls (Fig. 5b). These results indicate that AfuWetA is essential for both viability and integrity of conidia.

We then examined the effects of $\triangle A f u w e t A$ on spore tolerance to various stresses and found that $\triangle A$ fuwet $A$ mutant conidia exhibited drastically reduced tolerance to thermal (Fig. 5c), oxidative (Fig. 5d) and osmotic (Fig. 5e) stresses. As trehalose is essential for long-term spore viability and stress resistance (Fillinger et al., 2001; Ni \& Yu, 2007), we asked whether the $\Delta$ AfuwetA conidia contained a sufficient amount of trehalose, and therefore examined the amount of trehalose in 2-day-old conidia of WT, $\triangle A$ fuwet $A$ and complemented strains. As shown in Fig. 5(f), $\Delta A$ fuwet $A$ conidia did not contain any trehalose, whereas WT and complemented strains harboured about 3.4 pg trehalose per conidium, indicating that $A f u W e t A$ plays an essential role in trehalose biogenesis in conidia, which probably affects the viability and stress tolerance of conidia.

\section{AfuWetA functions in the completion of the conidial wall}

To examine the role of AfuWetA in conidial wall formation, WT and wetA mutant conidia in both $A$. 
nidulans and A. fumigatus were compared by TEM (Fig. 6a). The A. fumigatus WT strain exhibited similar conidial wall structure to A. nidulans (Sewall et al., 1990b). As shown in Fig. 6(a), WT conidia form the crenulated electron-dense $\mathrm{C} 1$ layer and the condensed electron-light $\mathrm{C} 2$ layer. In the wet $A$ mutants, although the $\mathrm{C} 1$ and $\mathrm{C} 2$ layers were formed, the $\mathrm{C} 1$ layer was not crenulated and the $\mathrm{C} 2$ layer failed to condense, resulting in a thicker conidial wall than in the WT strain. We then measured the conidial wall thickness of the test strains. The A. nidulans and A. fumigatus wetA mutant conidial walls had a mean thickness of 0.2 and $0.18 \mu \mathrm{m}$, respectively, while both WT conidia exhibited a mean thickness of $0.13 \mu \mathrm{m}$. These data indicate that the $\Delta$ AfuwetA mutant exhibits conidial wall defects similar to those found in A. nidulans, and that AfuWetA plays an essential role in conidial wall completion and spore maturation.

\section{AfuWetA is required for proper hyphal branching and timely conidiation}

Given that the AfuwetA gene is activated during the late stage of conidiation, and that its mRNA accumulates preferentially in mature conidia, we asked whether the absence of AfuwetA has any effect on conidial germination and early hyphal growth. We found that after $8 \mathrm{~h}$ incubation in liquid submerged culture, conidia of WT and complemented strains produced bi- or multi-directional germ tubes that formed branches which eventually turned into compact mycelial aggregates. However, in the $\Delta$ AfuwetA mutant, each conidium formed only one unidirectional germ tube without branching, which resulted in loosened mycelial aggregates (Fig. 6b). Moreover, after $14 \mathrm{~h}$ incubation, the $\triangle$ AfuwetA mutant clearly showed reduced branch density compared to the WT and complemented strains. The branching rates of these strains were defined by peripheral growth unit (PGU) values (Etxebeste et al., 2009; see Fig. $6 \mathrm{~b})$. The PGU values of WT, $\triangle A$ fuwetA and complemented strain hyphae were $18.75 \pm 1.56 \mu \mathrm{m}, 86.18 \pm 9.23 \mu \mathrm{m}$ and $19.27 \pm 2.39 \mu \mathrm{m}$, respectively, demonstrating an approximately fivefold reduction in hyphal branching in the $\triangle A f u w e t A$ mutant. These results suggest that AfuWetA is associated with proper germ-tube formation and vegetative growth. This is consistent with the finding that overexpression of wetA in hyphae causes excessive branching in A. nidulans (Marshall \& Timberlake, 1991).

We also investigated the effects of $\triangle A$ fuwetA on development. Conidia of WT, $\triangle$ AfuwetA and complemented strains were inoculated into liquid MMG, incubated for $18 \mathrm{~h}$ at $37{ }^{\circ} \mathrm{C}, 250$ r.p.m., and the mycelium was collected and transferred into liquid (L) and solid (S) MMG and $\mathrm{MMG}+0.6 \mathrm{M} \mathrm{KCl}$ and further incubated at $37{ }^{\circ} \mathrm{C}$ (250 r.p.m. for liquid culture). Developmental progression under each culture condition was examined. As shown in Fig. 6(c), while the WT and complemented strains readily produced abundant conidiophores when exposed to air (solid MMG) and/or high salt (solid/liquid MMG $+\mathrm{KCl}$ ), the $\triangle$ AfuwetA mutant exhibited delayed and reduced conidiation in all three inducing conditions. These results suggest that, although activated during the late phase of conidiation, $A f u W e t$ is necessary for the proper initiation and progression of development.

\section{Genetic interactions between conidiation genes in A. fumigatus}

To dissect the genetic interactions between the central regulatory genes in A. fumigatus, a series of Northern blot analyses were carried out. As shown in Fig. 7(a), the absence of AfubrlA eliminated the expression of all conidiation-specific genes tested, indicating that $A f u B r l A$ functions upstream of AfuabaA, AfuwetA, AfuvosA and AfurodA. As AfuAbaA is necessary for the activation of AfuwetA, AfuwetA mRNA accumulation was not detected in the $\triangle A$ fuabaA mutant, confirming the AfubrlA $\rightarrow$ AfuabaA $\rightarrow$ AfuwetA regulatory cascade in A. fumigatus. Moreover, levels of AfuvosA mRNA decreased considerably in both the $\triangle A f u a b a A$ and $\triangle A$ fuwetA mutants, indicating the requirement for both genes in proper AfuvosA expression. However, as the activation of AfuvosA occurs before the activation of AfuwetA in WT, it is proposed that AfuAbaA primarily activates AfuvosA with the assistance of AfuWetA. Importantly, AfubrlA is highly upregulated in $\triangle A$ fuaba $A$ and $\triangle A$ fuwet $A$ mutants, particularly in conidia (aberrant conidiophores in $\triangle A f u a b a A$ ) and early $(6 \mathrm{~h}$ ) and late $(24 \mathrm{~h})$ phases of vegetative growth, suggesting that $A f u A b a A$ and $A f u W e t A$ are necessary for proper negative feedback regulation of AfubrlA upon completion of conidiation and during certain phases of vegetative growth. AfuRodA, a small, secreted, moderately hydrophobic polypeptide, is involved in the formation of the outermost rodlet layer of conidia, and the conidia of AfurodA mutants lack their external rodlet layer and are hydrophilic (Thau et al., 1994). The observations that no AfurodA mRNA was detected in the $\triangle A$ fubrlA mutant and that levels of AfurodA were low in the $\triangle A$ fuabaA mutant suggest that $A f u B r l A$ and $A f u A b a A$ activated $A f u r o d A$ in a supportive manner. Taking all these observations together, a genetic model regulating asexual development in $A$. fumigatus is proposed (Fig. 7b).

\section{DISCUSSION}

While a number of key developmental regulators have been identified and characterized in A. nidulans, they remain to be studied in other aspergilli. In this study, we have characterized how AfuabaA and AfuwetA control growth and development of the opportunistic pathogen $A$. fumigatus and have demonstrated that central regulatory components are conserved in both Aspergillus species: AfuAbaA functions in the differentiation of phialides (the conidiogenous cells which are required for the generation of conidia), and $A f u$ WetA plays a role in the completion of the conidial wall and is essential for trehalose biogenesis, conidial viability, integrity and stress tolerance. 
(a)

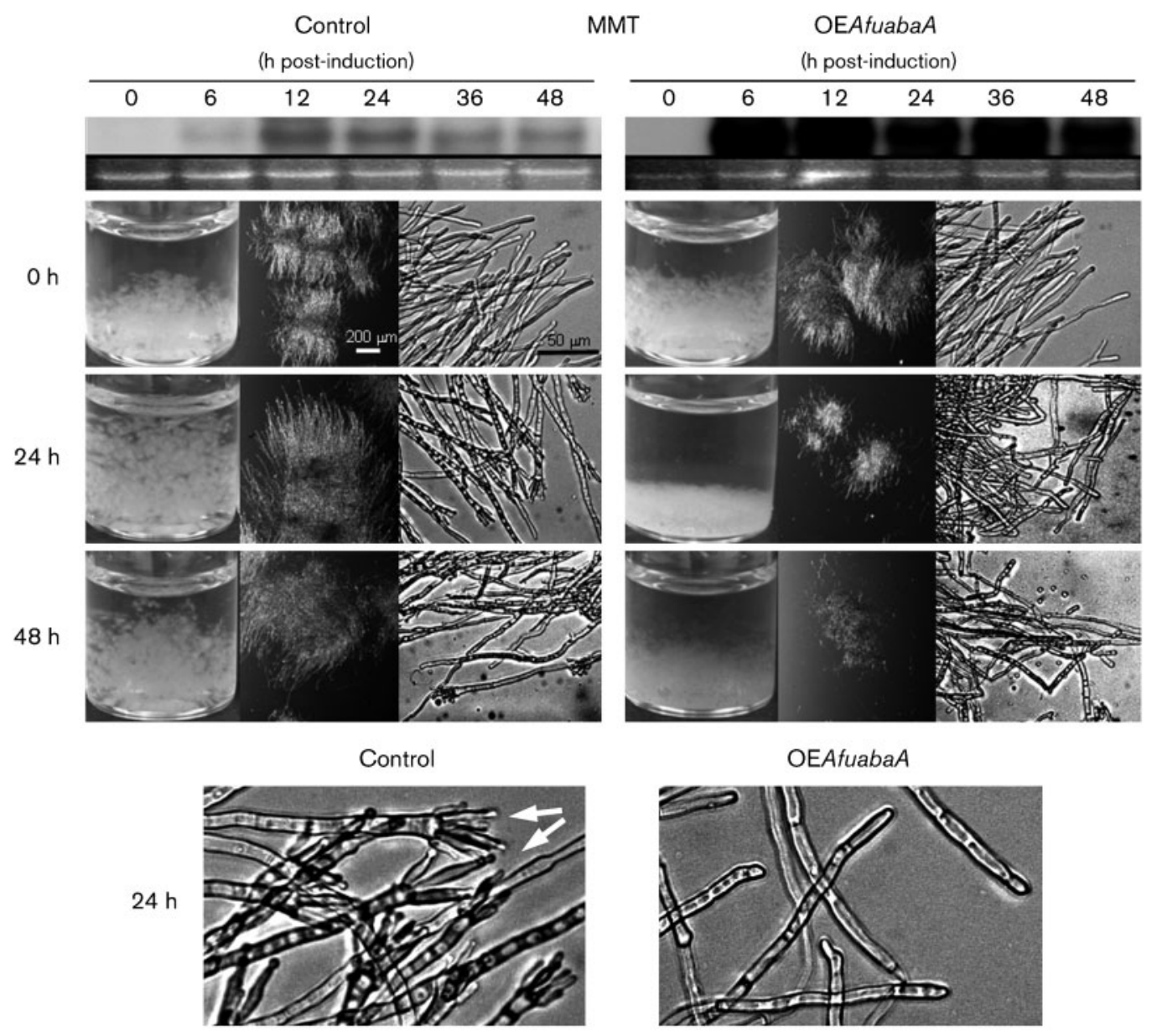

(b)
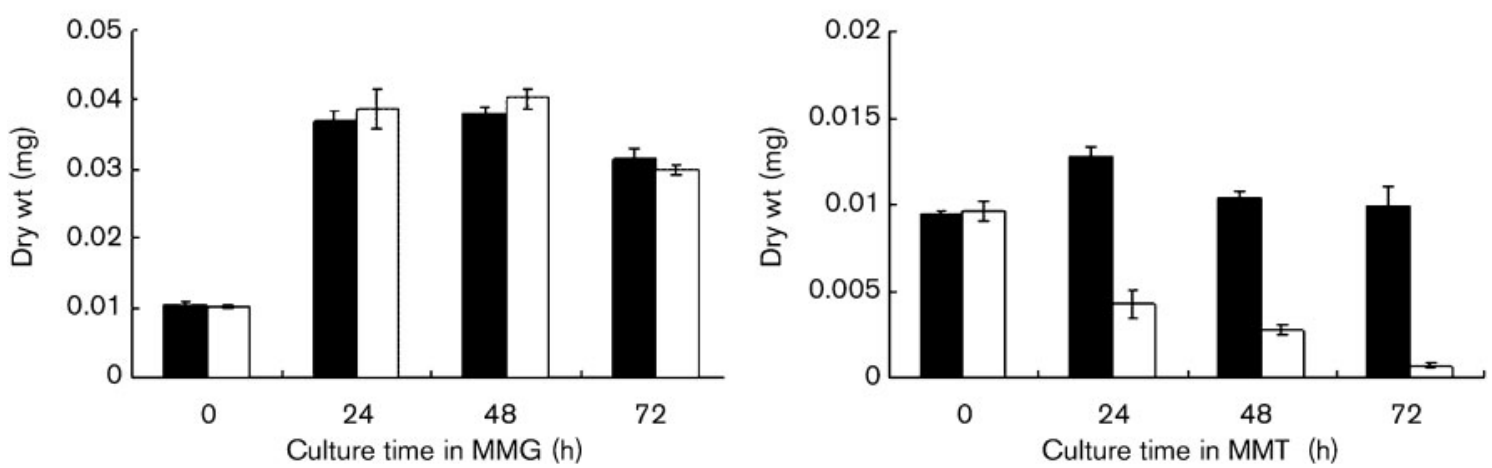

Fig. 4. Overexpression of $A f u a b a A$ accelerates autolysis and cell death, but inhibits growth and development. (a) Phenotypes of control (TLI11) and OEAfuabaA (TLI9) strains in liquid MMT submerged culture, and Northern blot analyses for levels of $A f u a b a A$ mRNA. Micrographs were taken at 0,24 and $48 \mathrm{~h}$ of incubation after transferring $18 \mathrm{~h}$-old vegetative cells into MMT. The close-up views show the conidiation defects in the OEAfuabaA strain. Arrows indicate the simplified conidiophores formed by the control strain at $24 \mathrm{~h}$. (b) Mycelial dry weight of control (black bars) and OEAfuabaA (white bars) strains grown in liquid MMG or MMT. (c) Cell viability of control and OEAfuabaA strains determined by $A B$ reduction rates. Data are the mean values of three individual cultures. Note that the OEAfuabaA strain exhibits reduced viability at $24 \mathrm{~h}$. Black bars, control in MMG; dotted bars, OEAfuabaA in MMG; grey bars, control in MMT; white bars, OEAfuabaA in MMT. (d) Phenotypes of control and OEAfuabaA strains grown on solid inducing medium (MMT) at $37{ }^{\circ} \mathrm{C}$ for 3 days, and the number of conidia per $\mathrm{mm}^{2}$ of control and OEAfuabaA colonies. Note that the OEAfuabaA strain formed small colonies with

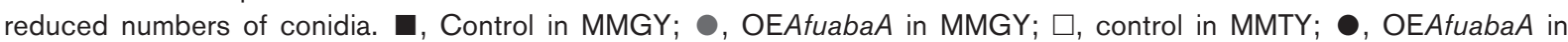
MMTY. 
(c)

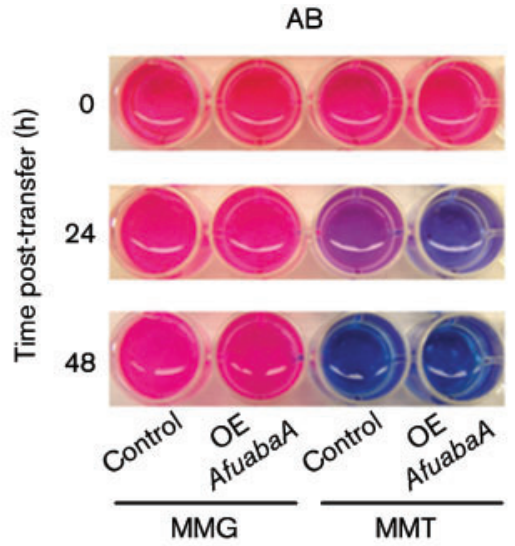

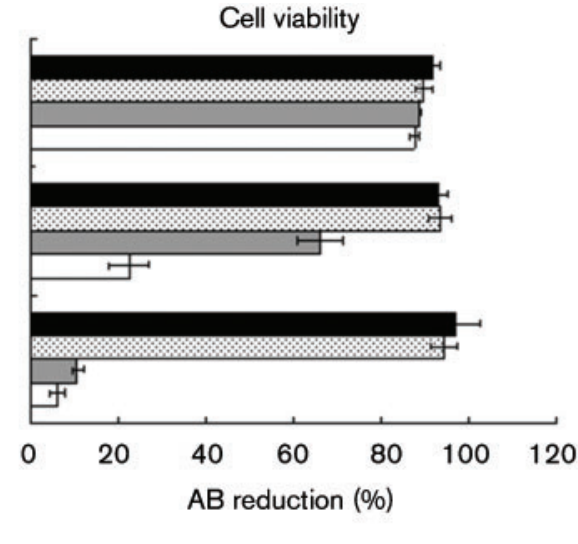

(d)

MMT

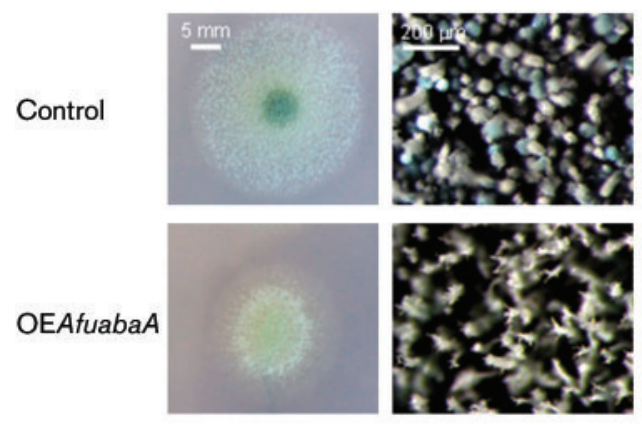

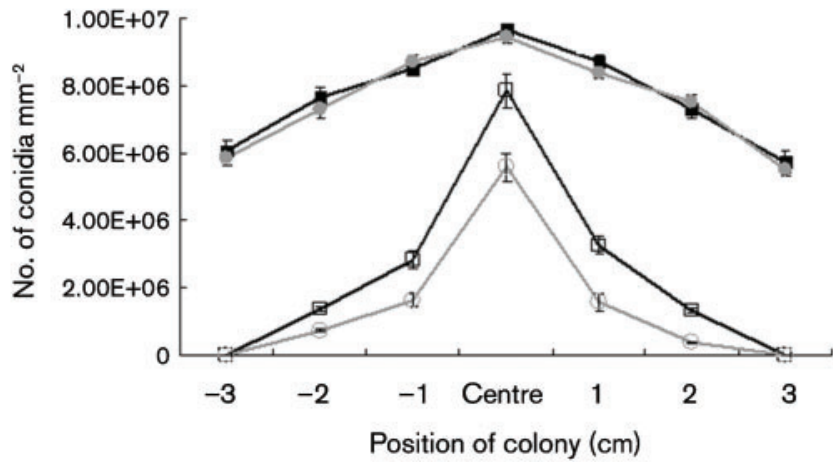

Importantly, AfuAbaA also plays a crucial role in cell death and autolysis in A. fumigatus. Autolysis can be defined as a natural process of self-digestion of aged hyphal cultures, occurring as a result of hydrolase activity, causing vacuolization and disruption of organelle and cell-wall structure (White et al., 2002). Previously, it has been reported that loss-of-function mutations in $a b a A$ of $A$. nidulans result in reduced and delayed hydrolase (chitinase) production (Emri et al., 2008). Thus it can be speculated that AfuAbaA affects the autolysis process potentially by activating production of various hydrolases. As the regulatory pathways of autolysis and apoptotic cell death are tightly coupled, the absence of AfuabaA also results in delayed cell death via an unknown mechanism. For the development of novel antifungal targets, it would be interesting to investigate the molecular

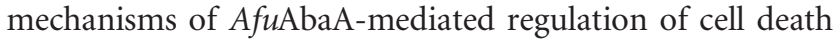
and autolysis and identify its potential target genes.

Probably the most important finding in our present study is that AfuwetA is essential for trehalose biogenesis in conidia. Trehalose is an important osmolyte produced by bacteria, fungi, insects and plants to protect the integrity of cells against various environmental stresses (reviewed by Elbein et al., 2003; Ni \& Yu, 2007). Thus, the absence of trehalose in fungal spores results in rapid loss of viability and extreme sensitivity to various stresses. In fact, as reported above, $\Delta$ AfuwetA conidia are highly sensitive to heat, salt and oxidative stresses. While our previous study reported that the deletion of AfuvosA resulted in a $50 \%$ reduction in trehalose in A. fumigatus conidia ( $\mathrm{Ni} \& \mathrm{Yu}$, 2007), this is the first report of the essential role of WetA in trehalose biogenesis in fungal conidia.

TEM analyses indicate that $\triangle$ AfuwetA conidia exhibit unclear and aberrant wall layers compared to those of WT, i.e. AfuWetA is required for the modification of conidial wall and the integrity of conidia, as reported in A. nidulans (Sewall et al., 1990b). In A. nidulans, conidium maturation (a differentiation process occurring after the formation of the septum between the phialide and the newly formed conidium) can be divided into three stages (Sewall et al., 1990b). At stage I, conidia are completely separated from the phialide and they contain two cell-wall layers, C1 (outer) and C2 (inner). At stage II, C2 condenses and C1 becomes crenulated. In the third stage, two new cell-wall layers, C3 and C4, are formed and render the conidia impermeable and mature. Loss-of-function mutations in the wetA locus lead to defects in the formation of the C3 and $\mathrm{C} 4$ wall layers and in condensation of the $\mathrm{C} 2$ wall layer, resulting in incomplete maturation and consequent lysis of conidia (Sewall et al., 1990b). The absence of AfuwetA results in almost identical defects in the formation of layers C3 and C4 and condensation of the C2 wall layer, suggesting that the spore-wall components affected by WetA might be conserved in both aspergilli.

Microscopical analyses of conidial germination in the $\triangle$ AfuwetA mutant revealed that most conidia form only 
(a)

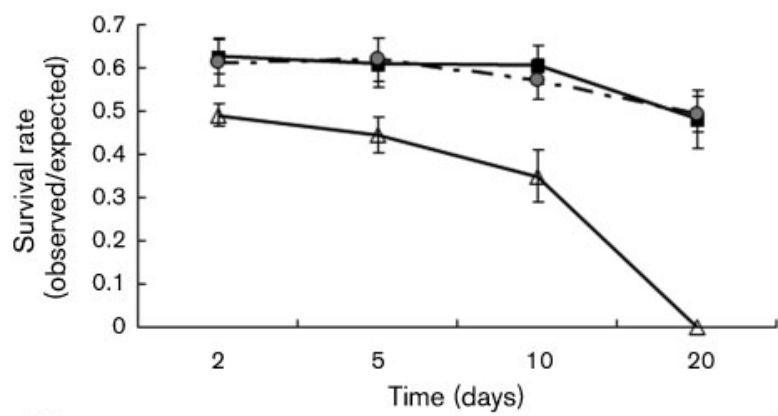

(c)

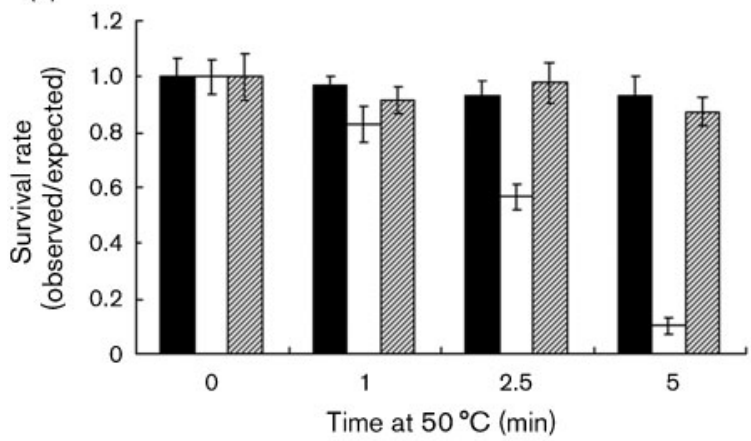

(b)

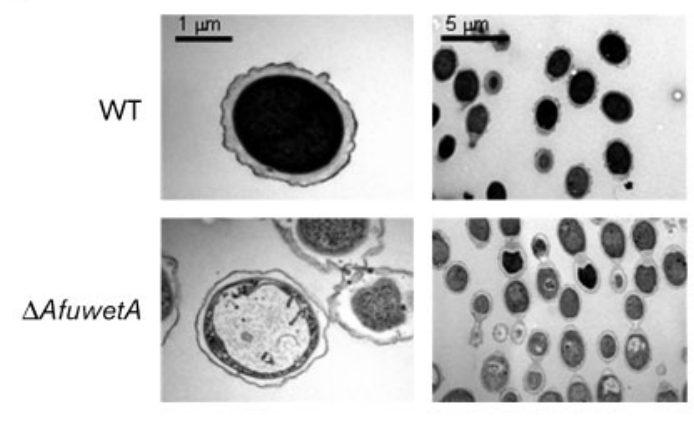

(d)

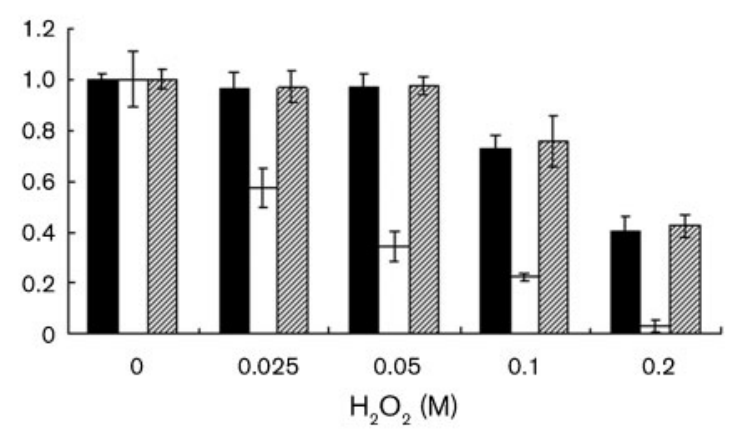

(f)

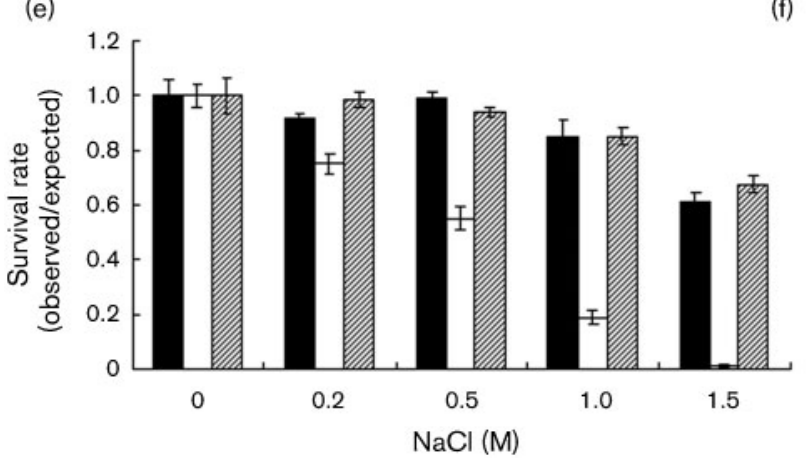

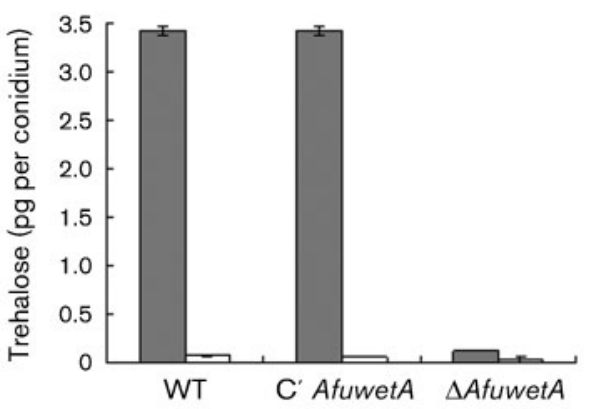

Fig. 5. Requirement of $A f u W e t A$ for viability and stress tolerance of conidia. (a) Viability of conidia of WT (Af293, $\mathbf{\square}), \Delta A f u w e t A$ (TSGw4, $\triangle$ ) and complemented (TLI4, O) strains grown at $37{ }^{\circ} \mathrm{C}$ for 2, 5, 10 and 20 days. (b) TEM images of 2-day-old conidia of WT and $\triangle$ AfuwetA strains. Note that even at day 2 , about $20 \%$ of the $\triangle A$ AuwetA conidia have lost their cytoplasm. (c-e) Tolerance of WT (black bars), $\triangle$ AfuwetA (white bars) and complemented strain (hatched bars) conidia to heat (c), oxidative (d) and salt (e) stresses. (f) The amount of trehalose (pg per conidium) in 2-day-old conidium of WT, $\triangle A f u w e t A$ and complemented strains. No trehalase treatment (white bars) served as a negative control.

one germ tube, and that the branching of the germlings is reduced. Due to the inhibited hyphal branching, the $\Delta$ AfuwetA mutant growing in submerged culture formed less compact mycelial aggregates than those of the WT. A previous study has proposed that branch formation involves hyphal wall remodelling accompanied by the accumulation of vesicles at the lateral wall (reviewed by Adams, 2004). Importantly, Marshall \& Timberlake (1991) reported that overexpression of $A$. nidulans wet $A$ in hyphae inhibited growth and caused excessive branching. This is somewhat consistent with our finding that $\Delta$ AfuwetA mutant hyphae show an approximately fivefold reduction in branching (Fig. 6b). These results imply that the absence of AfuwetA not only affects the modification of the conidial wall, but also causes hyphal wall alterations, which in turn affect hyphal branching.

A genetic model depicting the regulation of $A$. fumigatus conidiation is presented (Fig. 7b). In this model, upstream regulator $A f u$ FluG is proposed to activate $A f u f l b$ (e.g. $A f u f l b B$ and AfuflbE; Yu et al., 2006; Kwon et al., 2010; Xiao et al., 2010; $\mathrm{Yu}, 2011$ ) and $A f u B r l A$, thereby triggering the 
(a)
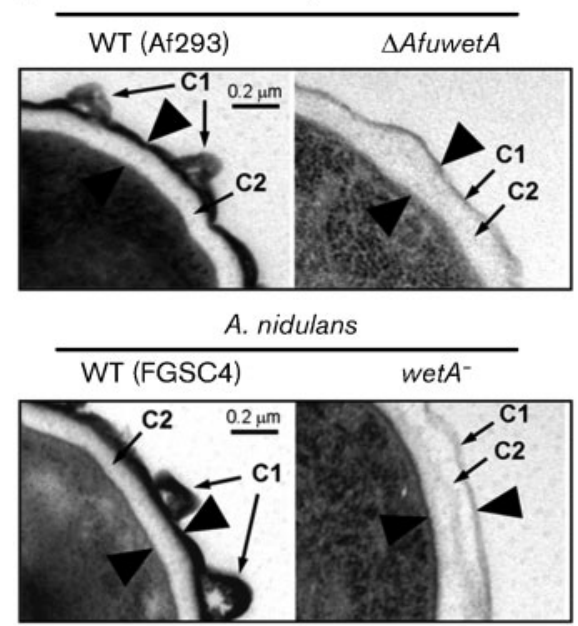

(c) (b)

$8 \mathrm{~h}$

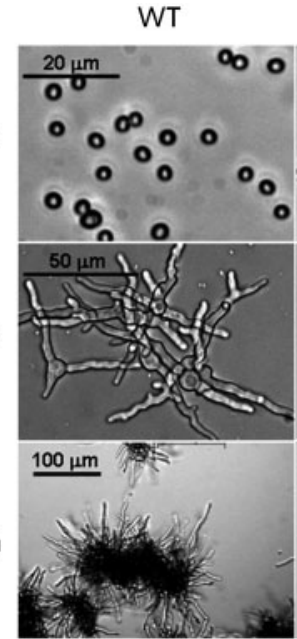

$18.75 \pm 1.56 \mu \mathrm{m}$ $\triangle$ AfuwetA

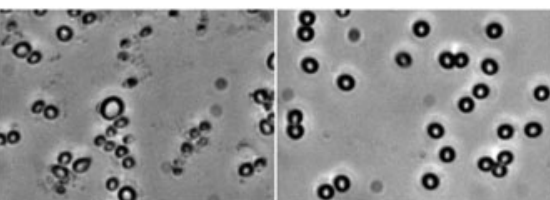

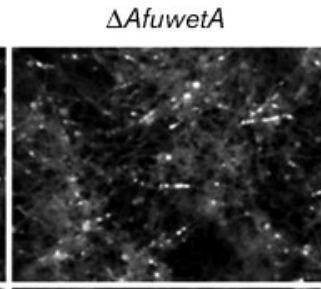

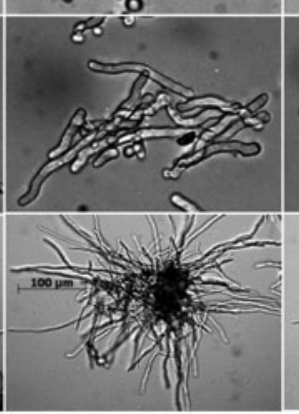

$86.18 \pm 9.23 \mu \mathrm{m}$

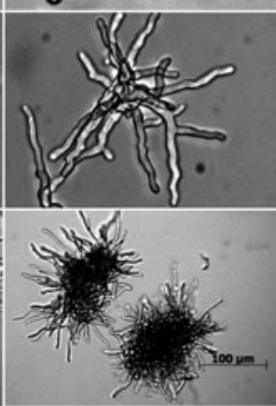

$19.27 \pm 2.39 \mu \mathrm{m}$

S

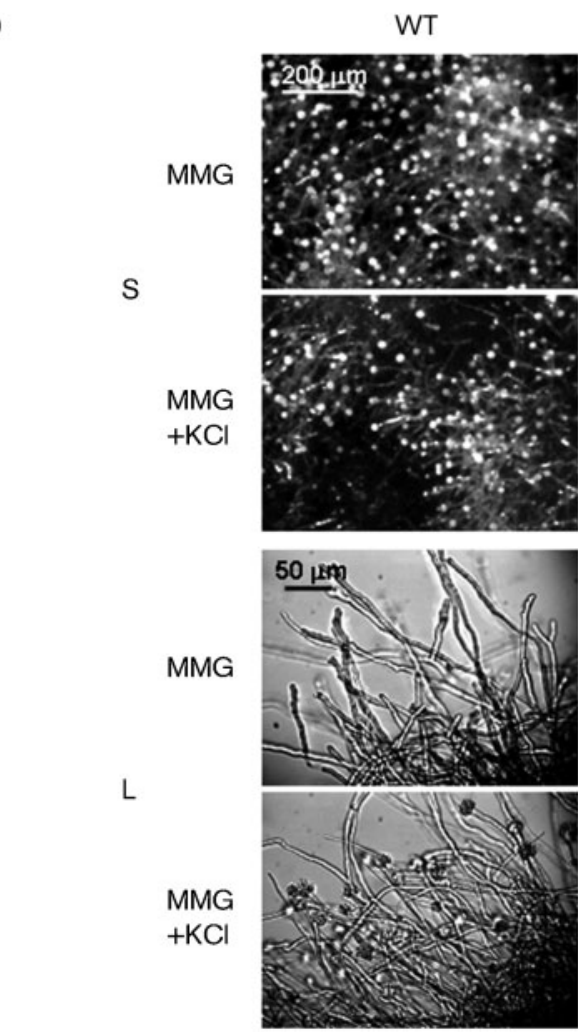

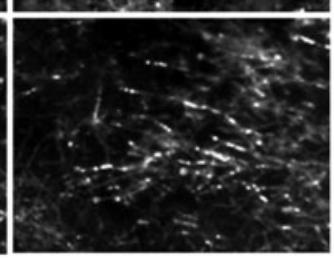
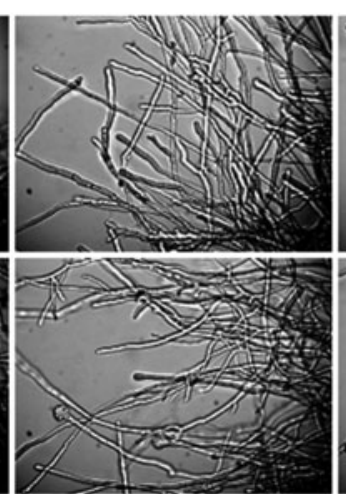

C' AfuwetA
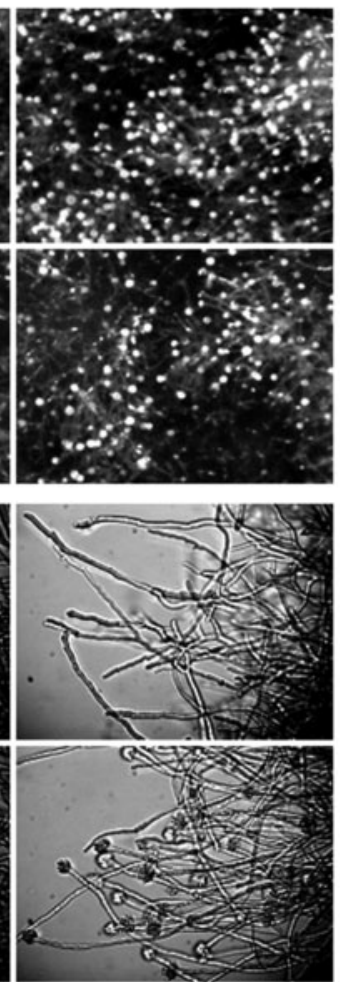

Fig. 6. Additional phenotypes resulting from $\triangle$ AfuwetA. (a) TEM images of 2-day-old conidia wall structures of $A$. fumigatus WT (Af293) and the $\triangle$ AfuwetA mutant (TSGw4), and $A$. nidulans WT (FGSC4) and the wet $A^{-}$mutant (AJC472.1). Arrowheads indicate the wall thickness in four conidia. $\mathrm{C} 1$ (outer) and $\mathrm{C} 2$ (inner) indicate the wall layers of $A$. nidulans conidia as reported by Sewall et al. (1990b). (b) Conidia of WT, $\triangle A f u w e t A$ and complemented strains were cultured in liquid MMG. Photomicrographs were taken at 8 and $14 \mathrm{~h}$. The PGU values are indicated below the photograph. (c) WT, $\triangle A f u w e t A$ and complemented strains grown under conditions that induce development. After $18 \mathrm{~h}$ incubation in liquid MMG, mycelia were transferred to both solid (S) and liquid (L) MMG and/or MMG $+\mathrm{KCl}$. Photomicrographs were taken after $6 \mathrm{~h}$ (solid medium, S) and $12 \mathrm{~h}$ (liquid medium, L) incubation. Note the delayed conidiation caused by the absence of AfuwetA.

central regulatory pathway $(A f u b r l A \rightarrow A f u a b a A \rightarrow A f u w e t A)$ of conidiation. Subsequently, AfuvosA (Ni \& Yu, 2007) is activated by $A f u A b a A$ with the assistance of $A f u$ WetA.
AfuWetA and AfuVosA coordinately complete spore maturation, including trehalose biogenesis. As found in A. nidulans, both $A f u B r l A$ and $A f u A b a A$ are required for 


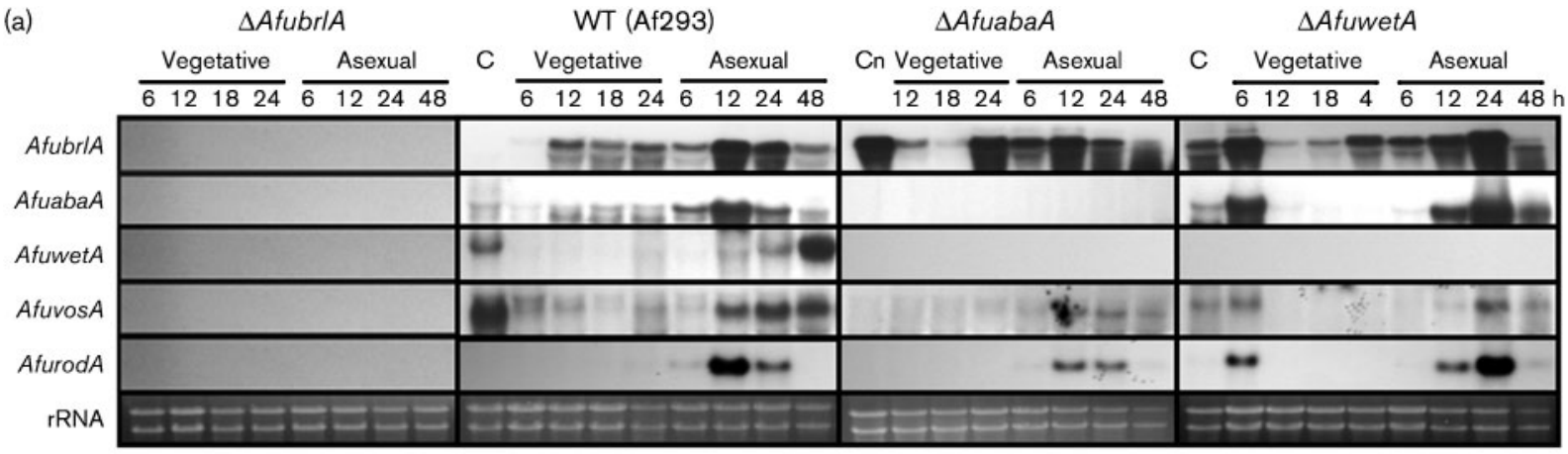

(b)

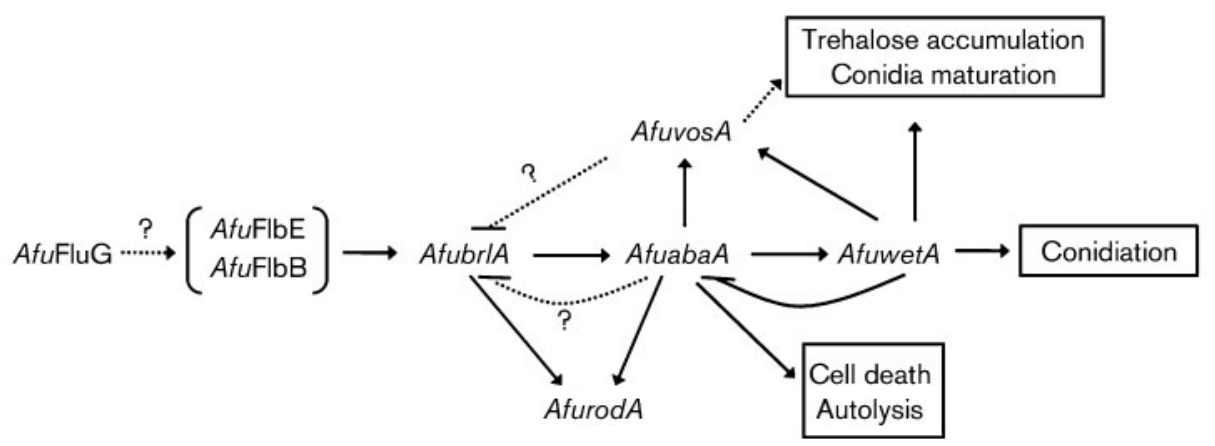

(c)

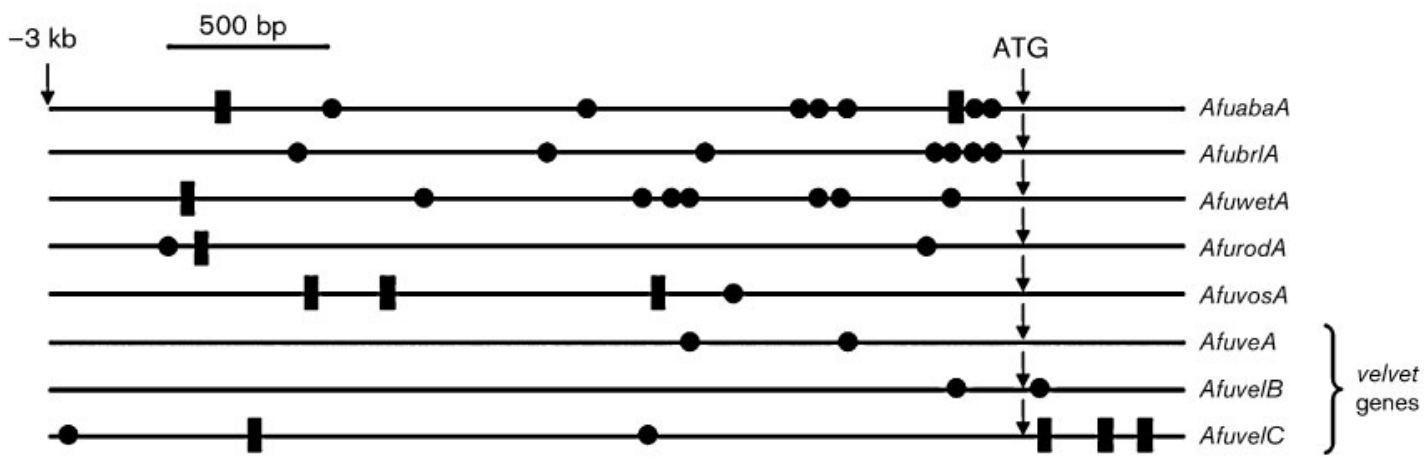

Fig. 7. Further expression studies and a model for developmental regulation in $A$. fumigatus. (a) Levels of various developmental genes (AfubrlA, AfuabaA, AfuwetA, AfuvosA and AfurodA) in four strains $\triangle$ AfubrlA (A1176), WT (Af293), $\triangle A f u a b a A$ (TSGa17) and $\triangle$ AfuwetA (TSGw4). C, conidia; Cn, conidiophore. (b) Model for regulation of $A$. fumigatus conidiation (see Discussion). (c) Positions of BREs $\left(\mathbf{\square}, 5^{\prime}-(\mathrm{C} / \mathrm{A})(\mathrm{G} / \mathrm{A}) \mathrm{AGGG}(\mathrm{G} / \mathrm{A})-3^{\prime}\right)$ and AREs $\left(\mathbf{0}, 5^{\prime}-\mathrm{CATTCY}-3^{\prime}\right)$ in selected regulatory genes in $A$. fumigatus.

proper expression of AfurodA (see Adams et al., 1998). The repressive roles of $A f u \mathrm{AbaA}$ and $A f u$ WetA on expression of AfubrlA may be attributed to AfuVosA (or other velvet regulators) functioning in a negative-feedback loop (Ni \& Yu, 2007; Yu, 2011; H.-S. Park \& J.-H. Yu, unpublished data). The observations that $A f u$ WetA may negatively regulate $A f u a b a A$ and that $A f u A b a A$ may directly repress AfubrlA are indicated. AfuAbaA plays a key role in autolysis and cell death.

Previous studies have identified the consensus binding sites for $A$. nidulans $\mathrm{BrlA}$ and $\mathrm{AbaA}$ [BrlA response elements (BREs), 5'-(C/A)(G/A)AGGG(G/A)-3'; AbaA response elements (AREs), $5^{\prime}$-CATTCY-3', where $\mathrm{Y}$ is a pyrimidine]
(Chang \& Timberlake, 1993; Andrianopoulos \& Timberlake, 1994). We analysed the promoter regions of several $A$. fumigatus conidiation-related genes for the presence of the predicted A. nidulans BREs or AREs. As shown in Fig. 7(c), AREs are present in all genes tested, including AfubrlA, AfuwetA, AfuvosA, AfurodA, AfuveA, AfuvelB and AfuvelC, suggesting that $A f u A b a A$ might bind to these genes and affect their expression. Among those tested, AfuVeA, $A f u V e l B$ and $A f u V e l C$ are the velvet proteins that are highly conserved in filamentous fungi (Ni \& Yu, 2007; Bayram et al., 2008). In A. nidulans, the velvet complex has been shown to control development and secondary metabolism (Bayram et al., 2008). AfuAbaA may also influence secondary metabolism by regulating expression of AfuveA, 
AfuvelB and AfuvelC. As one, two and three BREs are found in the AfuwetA, AfuabaA and AfuvosA genes, respectively, and one is found in AfurodA, AfuBrlA may control AfuwetA, AfuabaA, AfuvosA and AfurodA expression. Further studies to identify $A f u A b a A$ and $A f u$ WetA target genes in $A$. fumigatus are in progress.

\section{ACKNOWLEDGEMENTS}

The authors express special thanks to Shilpa Gadwal, Na Gao and Hee-Soo Park for technical assistance and Ellin Doyle for critically reviewing the manuscript. L.T. was supported by the Graduate Scholarship Program sponsored by China Scholarship Council (CSC) and the Ministry of Education of China. This work was supported by National Science Foundation (IOS-0640067, IOS-0950850) and UWMadison Food Research Institute grants to J.-H. Y.

\section{REFERENCES}

Adams, D. J. (2004). Fungal cell wall chitinases and glucanases. Microbiology 150, 2029-2035.

Adams, T. H., Boylan, M. T. \& Timberlake, W. E. (1988). brlA is necessary and sufficient to direct conidiophore development in Aspergillus nidulans. Cell 54, 353-362.

Adams, T. H., Wieser, J. K. \& Yu, J.-H. (1998). Asexual sporulation in Aspergillus nidulans. Microbiol Mol Biol Rev 62, 35-54.

Agarwal, R., Hazarika, B., Gupta, D., Aggarwal, A. N., Chakrabarti, A. \& Jindal, S. K. (2010). Aspergillus hypersensitivity in patients with chronic obstructive pulmonary disease: COPD as a risk factor for ABPA? Med Mycol 48, 988-994.

Al-Nasiry, S., Geusens, N., Hanssens, M., Luyten, C. \& Pijnenborg, R. (2007). The use of Alamar Blue assay for quantitative analysis of viability, migration and invasion of choriocarcinoma cells. Hum Reprod 22, 1304-1309.

Andrianopoulos, A. \& Timberlake, W. E. (1991). ATTS, a new and conserved DNA binding domain. Plant Cell 3, 747-748.

Andrianopoulos, A. \& Timberlake, W. E. (1994). The Aspergillus nidulans $a b a A$ gene encodes a transcriptional activator that acts as a genetic switch to control development. Mol Cell Biol 14, 2503-2515.

Bayram, Ö., Krappmann, S., Ni, M., Bok, J.-W., Helmstaedt, K., Valerius, O., Braus-Stromeyer, S., Kwon, N.-J., Keller, N. P. \& other authors (2008). VelB/VeA/LaeA complex coordinates light signal with fungal development and secondary metabolism. Science 320, 1504-1506.

Boylan, M. T., Mirabito, P. M., Willett, C. E., Zimmerman, C. R. \& Timberlake, W. E. (1987). Isolation and physical characterization of three essential conidiation genes from Aspergillus nidulans. Mol Cell Biol 7, 3113-3118.

Brookman, J. L. \& Denning, D. W. (2000). Molecular genetics in Aspergillus fumigatus. Curr Opin Microbiol 3, 468-474.

Chang, Y. C. \& Timberlake, W. E. (1993). Identification of Aspergillus brlA response elements (BREs) by genetic selection in yeast. Genetics 133, 29-38.

Clutterbuck, A. J. (1969). A mutational analysis of conidial development in Aspergillus nidulans. Genetics 63, 317-327.

Cockrill, B. A. \& Hales, C. A. (1999). Allergic bronchopulmonary aspergillosis. Annu Rev Med 50, 303-316.

Dagenais, T. R. T. \& Keller, N. P. (2009). Pathogenesis of Aspergillus fumigatus in invasive aspergillosis. Clin Microbiol Rev 22, 447-465.
d'Enfert, C. \& Fontaine, T. (1997). Molecular characterization of the Aspergillus nidulans treA gene encoding an acid trehalase required for growth on trehalose. Mol Microbiol 24, 203-216.

Ejzykowicz, D. E., Cunha, M. M., Rozental, S., Solis, N. V., Gravelat, F. N., Sheppard, D. C. \& Filler, S. G. (2009). The Aspergillus fumigatus transcription factor Ace2 governs pigment production, conidiation and virulence. Mol Microbiol 72, 155-169.

Elbein, A. D., Pan, Y. T., Pastuszak, I. \& Carroll, D. (2003). New insights on trehalose: a multifunctional molecule. Glycobiology 13, 17R-27R.

Emri, T., Molnár, Z., Szilágyi, M. \& Pócsi, I. (2008). Regulation of autolysis in Aspergillus nidulans. Appl Biochem Biotechnol 151, 211220.

Etxebeste, O., Herrero-Garcia, E., Araújo-Bazán, L., Rodríguez-Urra, A. B., Garzia, A., Ugalde, U. \& Espeso, E. A. (2009). The bZIP-type transcription factor FlbB regulates distinct morphogenetic stages of colony formation in Aspergillus nidulans. Mol Microbiol 73, 775789.

Fillinger, S., Chaveroche, M., van Dijck, P., de Vries, R., Ruijter, G., Thevelein, J. \& d'Enfert, C. (2001). Trehalose is required for the acquisition of tolerance to a variety of stresses in the filamentous fungus Aspergillus nidulans. Microbiology 147, 1851-1862.

Gao, X., Chen, L., Hu, G. \& Mei, H. (2010). Invasive pulmonary aspergillosis in acute exacerbation of chronic obstructive pulmonary disease and the diagnostic value of combined serological tests. Ann Saudi Med 30, 193-197.

Käfer, E. (1977). Meiotic and mitotic recombination in Aspergillus and its chromosomal aberrations. Adv Genet 19, 33-131.

Kwon, N.-J., Shin, K.-S. \& Yu, J.-H. (2010). Characterization of the developmental regulator FlbE in Aspergillus fumigatus and Aspergillus nidulans. Fungal Genet Biol 47, 981-993.

Mah, J.-H. \& Yu, J.-H. (2006). Upstream and downstream regulation of asexual development in Aspergillus fumigatus. Eukaryot Cell 5, 15851595.

Marshall, M. A. \& Timberlake, W. E. (1991). Aspergillus nidulans wetA activates spore-specific gene expression. Mol Cell Biol 11, 55-62.

Mims, C. W., Richardson, E. A. \& Timberlake, W. E. (1988). Ultrastructural analysis of conidiophore development in the fungus Aspergillus nidulans using freeze-substitution. Protoplasma 144, 132141.

Mirabito, P. M., Adams, T. H. \& Timberlake, W. E. (1989). Interactions of three sequentially expressed genes control temporal and spatial specificity in Aspergillus development. Cell 57, 859-868.

Ni, M. \& Yu, J.-H. (2007). A novel regulator couples sporogenesis and trehalose biogenesis in Aspergillus nidulans. PLoS ONE 2, e970.

Ni, M., Gao, N., Kwon, N.-J., Shin, K.-S. \& Yu, J.-H. (2010). Regulation of Aspergillus conidiation. In Cellular and Molecular Biology of Filamentous Fungi, pp. 559-576. Edited by K. A. Borkovich \& D. J. Ebbole. Washington, DC: American Society for Microbiology.

Nierman, W. C., Pain, A., Anderson, M. J., Wortman, J. R., Kim, H. S., Arroyo, J., Berriman, M., Abe, K., Archer, D. B. \& other authors (2005). Genomic sequence of the pathogenic and allergenic filamentous fungus Aspergillus fumigatus. Nature 438, 1151-1156.

Reyes, G., Romans, A., Nguyen, C. K. \& May, G. S. (2006). Novel mitogen-activated protein kinase MpkC of Aspergillus fumigatus is required for utilization of polyalcohol sugars. Eukaryot Cell 5, 19341940.

Rhodes, J. C. \& Askew, D. S. (2010). Aspergillus fumigatus. In Cellular and Molecular Biology of Filamentous Fungi, pp. 697-716. Edited by K. A. Borkovich \& D. J. Ebbole. Washington, DC: American Society for Microbiology. 
Seo, J.-A., Guan, Y. \& Yu, J.-H. (2003). Suppressor mutations bypass the requirement of $f l u G$ for asexual sporulation and sterigmatocystin production in Aspergillus nidulans. Genetics 165, 1083-1093.

Seo, J.-A., Guan, Y. \& Yu, J.-H. (2006). FluG-dependent asexual development in Aspergillus nidulans occurs via derepression. Genetics 172, 1535-1544.

Sewall, T. C., Mims, C. W. \& Timberlake, W. E. (1990a). abaA controls phialide differentiation in Aspergillus nidulans. Plant Cell 2, 731-739.

Sewall, T. C., Mims, C. W. \& Timberlake, W. E. (1990b). Conidium differentiation in Aspergillus nidulans wild-type and wet-white (wetA) mutant strains. Dev Biol 138, 499-508.

Shin, K.-S., Kwon, N.-J., Kim, Y.-H., Park, H.-S., Kwon, G.-S. \& Yu, J.-H. (2009a). Differential roles of the ChiB chitinase in autolysis and cell death of Aspergillus nidulans. Eukaryot Cell 8, 738-746.

Shin, K.-S., Kwon, N.-J. \& Yu, J.-H. (2009b). Gbetagamma-mediated growth and developmental control in Aspergillus fumigatus. Curr Genet 55, 631-641.

Szewczyk, E., Nayak, T., Oakley, C. E., Edgerton, H., Xiong, Y., Taheri-Talesh, N., Osmani, S. A. \& Oakley, B. R. (2006). Fusion PCR and gene targeting in Aspergillus nidulans. Nat Protoc 1, 3111-3120.

Tao, L., Gao, N., Chen, S. \& Yu, J.-H. (2010). The choC gene encoding a putative phospholipid methyltransferase is essential for growth and development in Aspergillus nidulans. Curr Genet 56, 283-296.

Thau, N., Monod, M., Crestani, B., Rolland, C., Tronchin, G., Latgé, J. P. \& Paris, S. (1994), rodletless mutants of Aspergillus fumigatus. Infect Immun 62, 4380-4388.
Wasylnka, J. A. \& Moore, M. M. (2003). Aspergillus fumigatus conidia survive and germinate in acidic organelles of A549 epithelial cells. J Cell Sci 116, 1579-1587.

White, S., Mclntyre, M., Berry, D. R. \& McNeil, B. (2002). The autolysis of industrial filamentous fungi. Crit Rev Biotechnol 22, 1-14.

Xiao, P., Shin, K.-S., Wang, T. \& Yu, J.-H. (2010). Aspergillus fumigatus $f l b B$ encodes two basic leucine zipper domain (bZIP) proteins required for proper asexual development and gliotoxin production. Eukaryot Cell 9, 1711-1723.

Xue, T., Nguyen, C. K., Romans, A., Kontoyiannis, D. P. \& May, G. S. (2004). Isogenic auxotrophic mutant strains in the Aspergillus fumigatus genome reference strain AF293. Arch Microbiol 182, 346353.

Yamazaki, H., Yamazaki, D., Takaya, N., Takagi, M., Ohta, A. \& Horiuchi, H. (2007). A chitinase gene, $c h i B$, involved in the autolytic process of Aspergillus nidulans. Curr Genet 51, 89-98.

Yu, J.-H. (2011). Regulation of development in Aspergillus nidulans and Aspergillus fumigatus. Mycobiology (in press).

Yu, J.-H., Hamari, Z., Han, K.-H., Seo, J.-A., Reyes-Domínguez, Y. \& Scazzocchio, C. (2004). Double-joint PCR: a PCR-based molecular tool for gene manipulations in filamentous fungi. Fungal Genet Biol 41, 973-981.

Yu, J.-H., Mah, J.-H. \& Seo, J.-A. (2006). Growth and developmental control in the model and pathogenic aspergilli. Eukaryot Cell 5, 15771584.

Edited by: A. H. Herrera-Estrella 